\title{
PERCEPÇÃO DE CUIDADORES DE CRIANÇAS OBESAS ACERCA DA OBESIDADE INFANTIL
}

PERCEPTION OF CAREGIVERS OF OBESE CHILDREN ABOUT PEDIATRIC OBESITY

PERCEPCIÓN DE CUIDADORES DE NIÑOS OBESOS SOBRE LA OBESIDAD PEDIÁTRICA

Brena Barreto Barbosa 1

Maria Soraia Pinto 2

Ana Vaneska Passos Meireles ${ }^{3}$

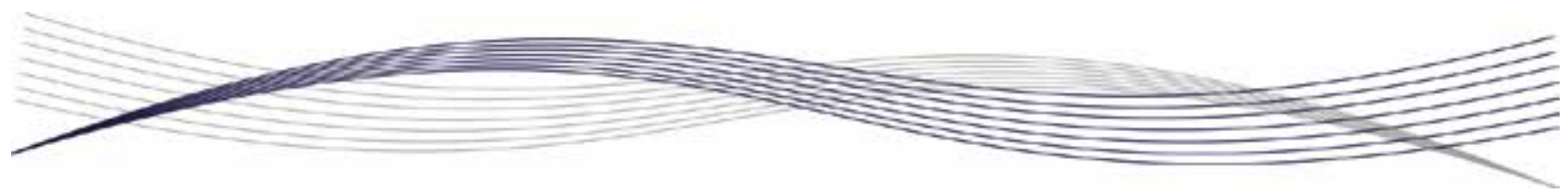

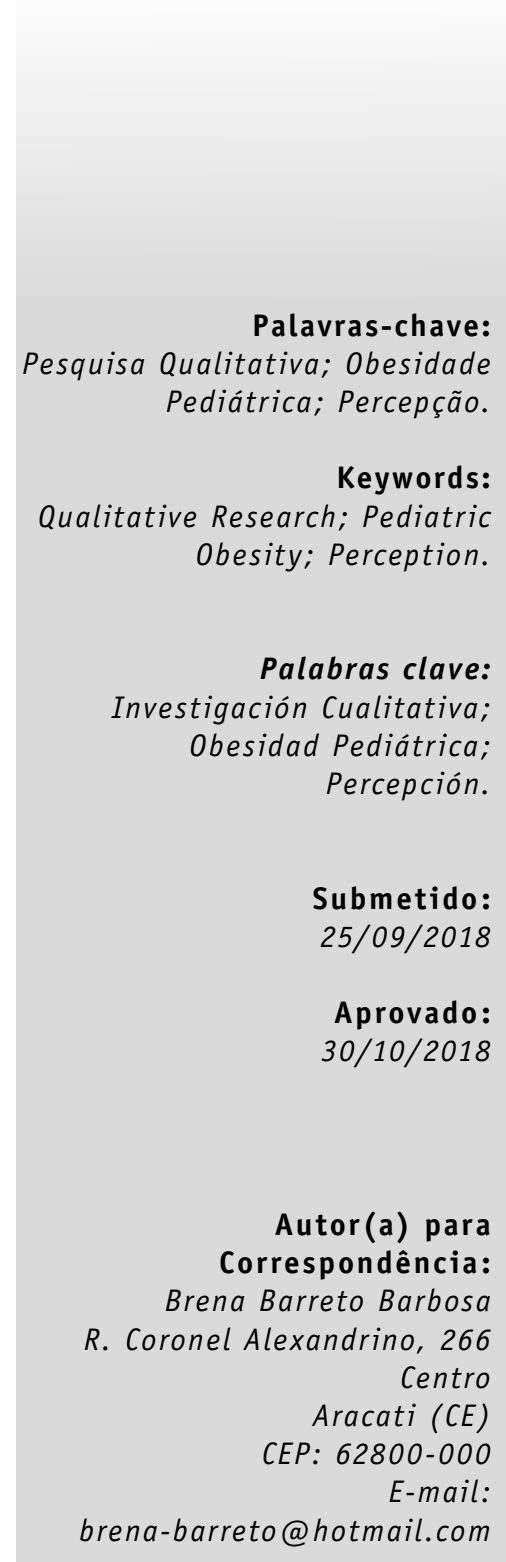

\section{RESUMO}

Este artigo tem por objetivo conhecer a percepção de cuidadores de crianças obesas acerca da obesidade infantil. Trata-se de pesquisa qualitativa realizada com mães de crianças menores de 5 anos que participam de um projeto multidisciplinar de combate à obesidade infantil. Os dados foram obtidos por meio de entrevista aberta ou em profundidade, registrada em áudio com o consentimento das participantes. Os resultados da pesquisa indicaram os três eixos temáticos que embasaram as linhas de análise (interpretação): a) "É... ela está muito gorda"; b) "Tropa das pernas"; e c) "Só sabe quem carrega". Constatou-se que as mães entrevistadas foram capazes de identificar a obesidade nas filhas, bem como de reconhecer algumas das consequências do excesso de peso. Além disso, elas associaram o peso saudável ao estereótipo da magreza.

\footnotetext{
1. Nutricionista. Aluna de Especialização em Saúde da Família e Comunidade na Escola de Saúde Pública do Ceará (ESP/CE). Residente em Saúde da Família e Comunidade. Acaraú (CE), Brasil.

2. Nutricionista. Aluna de Doutorado em Saúde Pública na Universidade Federal do Ceará (UFC). Mestre em Saúde Pública pela UFC. Professora da Universidade de Fortaleza (Unifor). Fortaleza (CE), Brasil.

3. Nutricionista. Mestre em Ciências Fisiológicas pela Universidade Estadual do Ceará (UECE). Professora da Unifor. Fortaleza (CE), Brasil.
} 


\section{ABSTRACT}

This article aims to know the perception of caregivers of obese children about childhood obesity. This is a qualitative research conducted with mothers of children under 5 years of age who participate in a multidisciplinary project to fight childhood obesity. Data was obtained through an open-ended or in-depth interview, recorded in audio with the participants' consent. The research results indicated the three thematic axes that supported the lines of analysis (interpretation): a) "Okay... she is very fat;" b) "Wobbly legs;" and c) "Only those carrying know about it." It was found that the mothers interviewed managed to identify obesity in their daughters, as well as recognize some of the consequences of overweight. Also, they associated healthy weight to the stereotype of thinness.

\section{RESUMEN}

Este artículo tiene como objetivo conocer la percepción de cuidadores de niños obesos sobre la obesidad pediátrica. Esta es una investigación cualitativa realizada con madres de niños menores de 5 años que participan en un proyecto multidisciplinario para combatir la obesidad pediátrica. Los datos se obtuvieron a través de una entrevista abierta o en profundidad, grabada en audio con consentimiento de las participantes. Los resultados de la investigación indicaron los tres ejes temáticos que apoyaron las líneas de análisis (interpretación): a) "Está bien... ella está muy gorda"; b) "Piernas temblorosas"; y c) "Sólo sabe quién lleva". Se constató que las madres entrevistadas lograron identificar la obesidad en sus hijas, asi como reconocer algunas de las consecuencias del sobrepeso. Además, ellas asociaron el peso sano al estereotipo de la delgadez.

\section{INTRODUÇÃ O}

A obesidade é identificada pelo acúmulo de tecido adiposo em uma região ou em todo o corpo. É considerada uma doença crônica e de múltiplas causas, resultando, na maioria das vezes, da combinação de fatores genéticos, ambientais e comportamentais ${ }^{1}$.

Atualmente, o excesso de peso é reconhecido como um problema de saúde pública, devido às proporções que vem atingindo ao longo dos anos e à sobrecarga que tem causado ao sistema de saúde por conta de suas complicações ${ }^{2}$. Como o excesso de peso na infância aumenta as chances de obesidade na vida adulta, as consequências dessa situação para as gerações futuras constituem motivo de preocupação ${ }^{3}$.

0 sobrepeso e a obesidade infantil vêm aumentando significativamente em todo o mundo desde a década de 1990 e têm atingido indivíduos em fases cada vez mais precoces da vida, sobretudo em países em desenvolvimento, onde a grande maioria dos afetados vive ${ }^{4}$.

Os números alarmantes sobre 0 aumento da obesidade em crianças se apresentam como reflexo do atual estilo de vida de grande parte da população infantil, que consiste em: consumo excessivo de alimentos com alta densidade energética, ricos em gorduras saturadas, açúcar e sal; falta de exercício físico; e predomínio de atividades sedentárias ${ }^{5}$.

Além dos dados biológicos, vale salientar que a obesidade precisa ser entendida como fenômeno multidimensional, ou seja: uma doença que abrange aspectos individuais e que pode ser vista como característica física, sendo, ainda, entendida, analisada e influenciada pela sociedade e pela cultura ${ }^{6}$.

A participação dos pais no tratamento e no acompanhamento da obesidade de seus filhos é fundamental para 0 sucesso das intervenções. 0 acompanhamento familiar deve promover hábitos saudáveis e apoio para lidar com as repercussões negativas da obesidade ${ }^{7}$. Tão importante quanto a sensibilização e a conscientização da família é a participação da escola e da sociedade no incentivo da promoção da saúde - como alimentação adequada e práticas de exercício físico ${ }^{8}$.

Como os comportamentos relacionados à saúde das crianças permanecem sob a influência e o controle dos seus pais, enfrentar a epidemia da obesidade infantil se mostra difícil e é necessário, portanto, aprimorar o entendimento das consequências desse problema de saúde entre os pais ${ }^{9}$.

Um desafio vital para a família de crianças obesas é proporcionar apoio e proteger a autoestima de seus filhos, ao mesmo tempo que se orienta adequadamente quanto às práticas alimentares mais apropriadas ${ }^{10}$. Assim, este artigo tem por objetivo conhecer a percepção de cuidadores de crianças obesas acerca da obesidade infantil. 


\section{METODOLOGIA}

Trata-se de estudo com abordagem qualitativa, de caráter exploratório e enfoque fenomenológico, realizado em Fortaleza (CE). Sua população consistiu em 8 mães de crianças menores de 5 anos, de ambos os sexos, que participam de um projeto multidisciplinar de combate à obesidade infantil em uma unidade básica de saúde (UBS). Os dados foram obtidos por meio de entrevista aberta ou em profundidade, registrada em áudio com o consentimento das participantes.

As entrevistas foram realizadas pela autora principal deste artigo, então aluna de Graduação em Nutrição, em único encontro com cada informante, em sala reservada no próprio espaço físico do projeto citado, propiciando maior privacidade e comodidade. As gravações foram transcritas na íntegra, de modo a documentar o testemunho das entrevistadas.

A pesquisa foi realizada em setembro e outubro de 2017. A coleta de dados cessou devido à saturação teórica ${ }^{11}$ dos achados nas entrevistas, resultante dos discursos convergentes que geraram as categorias analíticas. Para assegurar o anonimato dos sujeitos, os fragmentos dos testemunhos são identificados pela letra “E” (Entrevistada), seguida de sua ordenação numérica (E1 a E8).

A categorização do material indicou os três eixos temáticos que embasaram as linhas de análise (interpretação): a) "É... ela está muito gorda"; b) "Tropa das pernas"; e c) "Só sabe quem carrega".

Todas as participantes assinaram o termo de consentimento livre e esclarecido relativo ao estudo. Este foi aprovado pelo Comitê de Ética em Pesquisa da Universidade de Fortaleza (Unifor) sob o Parecer n. 2.234.850/2017, de acordo com os princípios da Resolução CNS n. 466/2012, que regulamenta a pesquisa com seres humanos no Brasil ${ }^{12}$.

\section{RESULTADOS E DISCUSSÃO}

As participantes têm idades entre 20 e 36 anos com média de 28 anos e todas elas relataram não estar empregadas, exercendo a função de donas de casa. Quanto à escolaridade, 6 entrevistadas tinham Ensino Fundamental incompleto e 2 tinham Ensino Médio incompleto.

Para sistematizar as informações das entrevistas gravadas e transcritas, a percepção das mães acerca da obesidade de seus filhos foi agrupada por categorias de unidades analíticas. Estas foram nomeadas ...mães de crianças

obesas tendem a

subestimar o peso

corporal de seus

filhos...

de acordo com as transcrições das entrevistas, ressaltando pontos subjacentes dos discursos: a) "É... ela está muito gorda"; b) "Tropa das pernas"; e c) "Só sabe quem carrega".

\section{"É... ela está muito gorda"}

Nesta primeira categoria empírica buscamos conhecer a percepção das mães sobre a obesidade de seus filhos. Quando indagadas sobre o peso da criança, todas as mães afirmaram que seus filhos estavam acima do peso.

Diferentemente de estudos recentes demonstrando que as mães de crianças obesas tendem a subestimar o peso corporal de seus filhos ${ }^{13}$ e apresentar uma percepção considerada distorcida, levando o excesso de peso a ser visto como normal ${ }^{14}$, as entrevistadas reconheceram a obesidade nas filhas, como indica a seguinte fala:

$$
\text { É... ela tá muito gorda. (E2) }
$$

Outro fator do discurso das entrevistadas que diverge de outros estudos sobre o tema é a consciência do risco apresentado pelo estado nutricional das filhas. Pesquisas recentes revelam que muitos pais de crianças com excesso de peso não o consideram um problema de saúde ${ }^{15}$.

[...] a gente tem que cuidar do peso, né? Senão vai ter só uma pressão alta, uma diabete. (E6)

Vale ressaltar que o cenário do estudo predispõe à percepção da obesidade, uma vez que as mães levaram seus filhos ao ambulatório exatamente para ter acesso ao tratamento da obesidade infantil.

0 s depoimentos indicam o desejo das mães de ver as filhas sem excesso de peso, além de demonstrarem associação entre peso saudável e corpo magro: 
[Resposta da mãe ao ser indagada sobre o que entende por peso saudável] Era se ela fosse mais magrinha, né? Era mais melhor. (E2)

A intenção de ver as filhas com um corpo magro pode decorrer da necessidade de aceitação imposta pela sociedade - muitas vezes, o corpo obeso é considerado anormal. Desejar um corpo magro para a filha seria uma maneira de evitar sofrimento nos relacionamentos e dificuldade nas interações sociais ${ }^{16}$.

Em estudo com o objetivo de conhecer a percepção da família de crianças obesas e da própria criança em relação ao seu corpo, observou-se que o excesso de peso trouxe prejuízo aos relacionamentos das crianças e à sua autoimagem, além da sensação de inadequação ao meio social em que estão inseridas ${ }^{17}$.

Percebeu-se que pode ocorrer reinterpretação da opinião dos outros acerca da obesidade das filhas, de modo a amenizar a situação e torná-la mais tolerável para ambas as partes:

[Resposta da mãe ao ser indagada sobre como se sente quando outras pessoas dizem que sua filha é "gordinha"] Eu não gosto muito, mas eu não... eu não reajo dessa maneira pra ela, eu digo só assim: "Mãe, quando di[ss]er que a minha filha é gordinha, a minha filha diz assim, ó: 'eu sou gordinha mas sou gostosa, eu sou gordinha mas sou importante pra minha mãe'". (E8)

Nota-se na fala da mãe a intenção de aconselhar a filha quanto à ressignificação da opinião de outrem acerca de sua aparência. Isso se manifesta como forma de proteção contra situações de preconceito que podem ser enfrentadas pela filha em decorrência de seu excesso de peso; além do estigma, tais situações incluem questões fisiológicas - como ilustra a próxima categoria.

\section{...o excesso de peso trouxe prejuizo aos relacionamentos das crianças e à sua autoimagem...}

\section{"Tropa das pernas"}

$\mathrm{Na}$ segunda categoria empírica abordamos as consequências da obesidade para as crianças, na percepção de suas mães, bem como as possíveis causas da obesidade infantil.

A obesidade causa grande impacto no sistema musculoesquelético das crianças ${ }^{18}$. Tal fato foi apontado nas falas, sendo reconhecido pelas entrevistadas como motivo de preocupação:

[...] eu me preocupo mais por causa das pernas dela. Por causa do peso que fica, os médicos disse [ram] que ela não se ajeita por causa do peso. (E1)

A preocupação das mães com os efeitos que a obesidade pode causar nas filhas pareceu refletirse principalmente na questão da aparência física das pernas das crianças. Isso pode justificar-se por ser uma manifestação visível das consequências da obesidade, sendo considerada prioritária em comparação com as manifestações assintomáticas da doença.

Algumas adaptações posturais em crianças, por exemplo, mudanças no alinhamento e na sustentação dos quadris, joelhos, tornozelos e pés, costumam ocorrer com vistas a suportar o excesso de peso e acabam provocando dores nos membros inferiores, desconforto e diminuição da capacidade de locomoção ${ }^{19}$ :

Ela é tropa das pernas. Se [andar] muito, ela cai. (E3)

É importante salientar que, além das consequências no sistema musculoesquelético, a obesidade infantil predispõe a várias complicações de saúde, como problemas respiratórios, diabetes mellitus, hipertensão arterial e dislipidemias, aumentando a incidência de morte na vida adulta. Ademais, crianças com obesidade estão expostas a relevante estresse psicológico devido ao estigma social ${ }^{20}$.

observou-se a obesidade como estigma no discurso das mães - com frequência associada a alta carga emocional:

Já hoje teve duas [crianças] que arengou com ela, que viu que não era do nível dela. [...] Acharam que ela causa medo, eu disse: "Não, ela é uma bebê, né?". (E2) 
Os obesos costumam ser estigmatizados porque não conseguem realizar atividades triviais com a mesma facilidade que outras pessoas ou por ser alvo de chacota (devido à sua imagem diferente) ${ }^{21}$.

\section{"Só sabe quem carrega"}

As entrevistadas reforçaram a dificuldade de cuidar de uma criança obesa, relatando principalmente 0 esforço para carregar as filhas no colo:

Mulher, porque ó, pra carregar ela no braço, ela pesa demais, tá entendendo?

Desde o momento do parto, é frequente que as mães carreguem seus filhos no colo durante longos períodos, até que eles possam iniciar sua marcha independente ${ }^{22}$. Tal hábito é uma das principais causas de lesão na coluna lombar e nos joelhos ${ }^{23}$, muitas vezes negligenciado pelas mães, que costumam menosprezar as dores e não buscam orientações sobre a postura adequada para prevenir tais lesões ${ }^{24}$.

Eu não aguento andar muito tempo com ela no colo, só se for caso de urgência. (E3)

0 discurso das mães acerca da obesidade pareceu centrar-se principalmente nas consequências do excesso de peso para a saúde das filhas, não sendo citadas preocupações relacionadas ao seu próprio bem-estar. 0s familiares parecem organizar-se em torno da obesidade da criança, comprometendo as relações sociais dos demais membros da família ${ }^{25}$.

A sobrecarga das mães de crianças obesas inclui não só a questão física, citada anteriormente, mas também a questão social, pois as mulheres exercem múltiplas funções e nem sempre podem dedicar-se com exclusividade ao tratamento da obesidade das filhas:

Cuido de toda [a] casa e de toda a família. [...] minha mãe disse, eu sou o pau da casa, se eu quebrar a casa cai. (E1)

Vale destacar que a responsabilidade pelo tratamento da obesidade infantil não deve resultar na culpabilização do indivíduo, da família ou mesmo da mãe. É necessário contemplar todo o círculo familiar e a comunidade em que a criança está inserida, de modo que as mudanças sejam

\section{A sobrecarga das \\ mães de crianças obesas inclui \\ não só a questão \\ física...}

acompanhadas por novos significados relativos à alimentação, ao corpo e ao viver ${ }^{26}$.

\section{CONCLUSÃO}

As mães entrevistadas se mostraram capazes de identificar a obesidade nas filhas, bem como de reconhecer algumas das consequências do excesso de peso. Além disso, elas associaram o peso saudável ao estereótipo da magreza, situação ainda muito comum devido à influência social.

No que concerne às repercussões da obesidade das filhas entre as mães, a sobrecarga física de carregar as crianças no colo foi amplamente citada, além das questões familiares que envolvem o cuidado de uma criança obesa por parte de mães que exercem múltiplas funções.

Constatou-se a necessidade de maior participação dos pais no acompanhamento das mudanças durante o tratamento da obesidade das filhas. Apesar das sessões destinadas às entrevistas serem abertas a quaisquer cuidadores de crianças neste estudo, apenas as mães estiveram presentes. Assim, uma sugestão para futuros estudos é investigar os motivos da falta de participação dos pais nos cuidados de saúde dos filhos, para proporcionar uma visão mais ampla da percepção dos cuidadores de crianças obesas acerca da obesidade infantil.

\section{CONTRIBUIÇÃO DAS AUTORAS}

Brena Barreto Barbosa contribuiu com a realização da pesquisa, o delineamento do estudo e a redação do manuscrito. Maria Soraia Pinto e Ana Vaneska Passos Meireles contribuíram com o delineamento do estudo e a redação e revisão crítica do manuscrito. 


\section{REFERÊNCIAS}

1. Sociedade Brasileira de Pediatria. Obesidade na infância e adolescência: manual de orientação. 2. ed. São Paulo: SBP; 2012.

2. Mendes EV. As redes de atenção à saúde. Brasília (DF): Organização Pan-Americana da Saúde; 2011.

3. Starc G, Strel J. Tracking excess weight and obesity from childhood to young adulthood: a 12year prospective cohort study in Slovenia. Public Health Nutr. 2011;14(1):49-55.

4. De Onis M, Blössner M, Borghi E. Global prevalence and trends of overweight and obesity among preschool children. Am J Clin Nutr. 2010;92(5):125764.

5. Moreno LA, Rodríguez G, Fleta J, Bueno-Lozano M, Lázaro A, Bueno G. Trends of dietary habits in adolescents. Crit Rev Food Sci Nutr. 2010;50(2):10612.

6. Pinto MS, Bosi MLM. Muito mais do que pe(n)sam: percepções e experiências acerca da obesidade entre usuárias da rede pública de saúde de um município do Nordeste do Brasil. Physis (Rio J) [serial on the internet]. 2010 [cited 2018 Nov 5];20(2):443-57. Available from: http://www.scielo.br/pdf/physis/ v20n2/a06v20n2.pdf

7. Vitorino SVZ, Soares LG, Marcon SS, Higarashi IH. Viver com obesidade infantil: a experiência de crianças inscritas em programa de acompanhamento multidisciplinar. Rev RENE [serial on the internet]. 2015 [cited 2018 Nov 5];15(6):980-9. Available from: $\quad$ http://periodicos.ufc.br/rene/article/ view $/ 3295 / 2534$

8. Lira RCM, Antunes LR, De Mesquita K0. Experiência de abordagem sobre alimentação entre crianças. Sanare (Sobral, Online) [serial on the internet]. 2016 [cited 2018 Nov 5];15(1):69-73. Available from: https://sanare.emnuvens.com.br/sanare/article/ view/930/559

9. Aparício G, Cunha M, Duarte J, Pereira A. Olhar dos pais sobre o estado nutricional das crianças préescolares. Millenium [serial on the internet]. 2011 [cited 2018 Nov 5];20(40):99-113. Available from: file:///D:/Dialnet-OLharDosPaisSobre0EstadoNutrici onalDasCriancasPree-4049644.pdf

10. Melo VLC, Serra PJ, Cunha CF. Obesidade infantil: impactos psicossociais. Rev Méd Minas Gerais [serial on the internet]. 2010 [cited 2018 Nov 5];20(3):36770. Available from: file:///D:/v20n3a14.pdf

11. Pires AP. Amostragem e pesquisa qualitativa: ensaio teórico e metodológico. In: Poupart J,
Deslauriers JP, Groulx LH, Lapemère A, Mayer R, Pires $A P$, organizers. A pesquisa qualitativa: enfoques epistemológicos e metodológicos. Petrópolis (RJ): Vozes; 2008. p. 154-211.

12. Brasil. Resolução n. 466, de 12 de dezembro de 2012. Diário Oficial da União, Brasília (2013 Jun 13); Sec 1.

13. Francescatto C, Santos NS, Coutinho VF, Costa RF. Mothers' perceptions about the nutritional status of their overweight children: a systematic review. J Pediatr [serial on the internet]. 2014 [cited 2018 Nov 5];90(4):332-43. Available from: http:// www.scielo.br/pdf/jped/v90n4/pt 0021-7557jped-90-04-00332.pdf

14. Camargo APPD, Barros Filho ADA, Antonio, MÂRD, Giglio JS. A não percepção da obesidade pode ser um obstáculo no papel das mães de cuidar de seus filhos. Ciênc Saúde Colet [serial on the internet]. 2013 [cited 2018 Nov 5];18(2):323-33. Available from: http://repositorio.unicamp.br/bitstream/ REPOSIP/200462/1/pmed 23358758.pdf

15. Tenorio A, Cobayashi F. Obesidade infantil na percepção dos pais. Rev Paul Pediatr [serial on the internet]. 2011 [cited 2018 Nov 5];29(4):63439. Available from: http://www.scielo.br/pdf/rpp/ v29n4/25.pdf

16. Oliveira JAN, Barreto JD, Mello A0, Freitas MCS, Fontes GAV. Percepção dos obesos sobre o discurso do nutricionista: estudo de caso. In: Freitas MCS, Fontes GAV, Oliveira JAN, organizers. Escritas e narrativas sobre alimentação e cultura. Salvador: Ed. UFBA; 2008. p. 175-189.

17. Venturini LP. Obesidade e família: uma caracterização de famílias de crianças obesas e a percepção dos familiares e das crianças de sua imagem corporal [thesis]. São Paulo: Universidade de São Paulo; 2000 [cited 2018 Nov 5]. Available from: file:///D:/TESE MESTRADO LUCIANA PETENUSCI VENTURINI.pdf

18. Wearing SC, Henning EM, Byrne MN, Steele JR, Hills AP. The impact of childhood obesity on musculoskeletal form. Obes Rev. 2006;7(2):209-18.

19. Bradalize M, Leite N. Alterações ortopédicas em crianças e adolescentes obesos. Fisioter Mov [serial on the internet]. 2010 [cited 2018 Nov 5];23(2):28388. Available from: http://www.scielo.br/pdf/fm/ v23n2/11.pdf

20. Adami F, Vasconcelos FAG. Childhood and adolescent obesity and adult mortality: a systematic review of cohort studies. Cad Saúde Pública [serial on the internet]. 2008 [cited 2018 Nov 5];24(Suppl 4):s558-s568. Available from: http://www.scielo.br/ $\mathrm{pdf} / \mathrm{csp} / \mathrm{v} 24 \mathrm{~s} 4 / 08 . \mathrm{pdf}$ 
21. Mattos RS, Luz MT. Sobrevivendo ao estigma da gordura: um estudo socioantropológico sobre obesidade. Physis (Rio J) [serial on the internet]. 2009 [cited 2018 Nov 5];19(2):489-507. Available from: $\quad$ http://www.scielo.br/pdf/physis/v19n2/ v19n2a14.pdf

22. Watson JC, Payne RC, Chamberlain AT, Jones RK, Sellers WI. The energetic costs of load-carrying and the evolution of bipedalism. J Hum Evol. $2008 ; 54(5): 675-83$.

23. Knapik J, Reynolds K, Staab J, Vogel JA, Jones B. Injuries associated with strenuous road marching. Mil Med. 1992;157(2):64-7.

24. Almeida LMS, Vasconcelos RS, Sales RP, Matos WLS, Viana SMN, Andrade EA, et al. Prevalência de algias da coluna vertebral em mães de crianças com paralisia cerebral não deambulantes. Revista de Saúde Pública de Santa Catarina [serial on the internet]. 2012 [cited 2018 Nov 5];5(1):28-37. Available from: http://revista.saude.sc.gov.br/ index.php/inicio/article/view/103/152

25. Nunes MCA, De Morais NA. As relações familiares de crianças obesas: uma análise dos discursos maternos. Clínica \& Cultura [serial on the internet]. 2012 [cited 2018 Nov 5];1(1):68-82. Available from: https://seer.ufs.br/index.php/clinicaecultura/ article/view/638/556

26. Brasil. Estratégia Intersetorial de Prevenção e Controle da Obesidade: recomendações para estados e municípios. Brasília, DF: Câmara Interministerial de Segurança Alimentar e Nutricional; 2014.
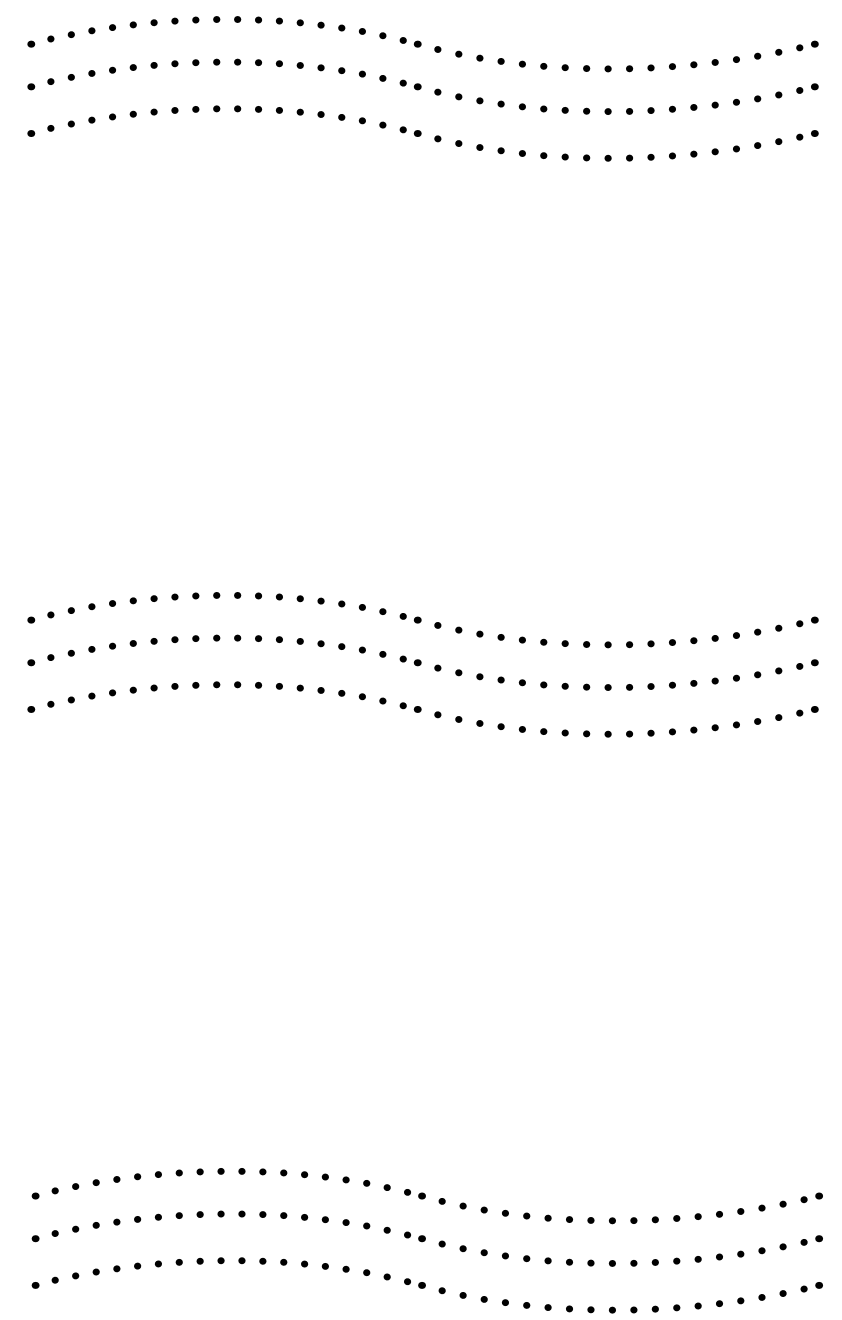
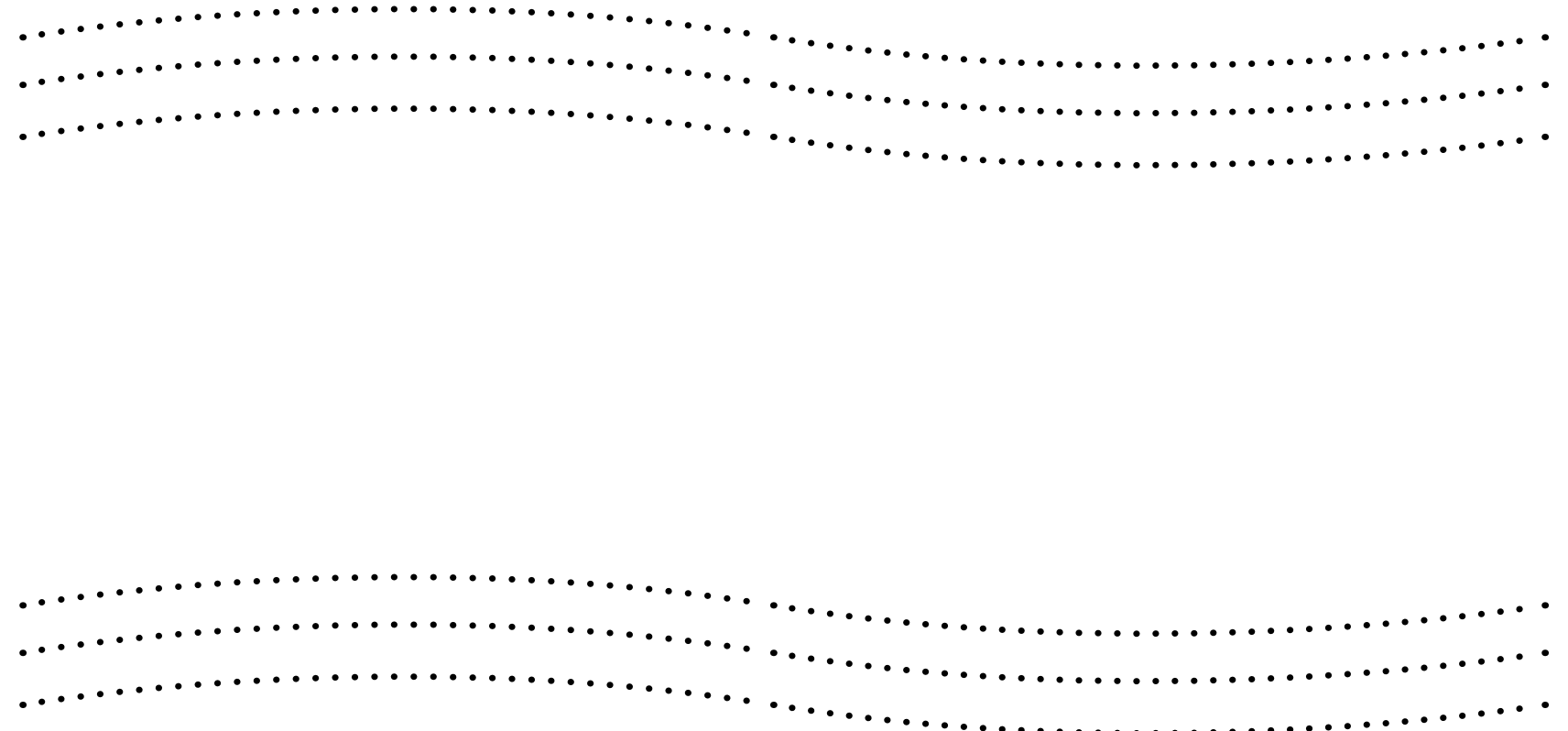